Bull. Austral. Math. Soc.

VOL. 73 (2006) [461-472]

\title{
ON THE LENGTHS OF PAIRS OF COMPLEX MATRICES OF SIZE AT MOST FIVE
}

\author{
W.E. Longstaff, A.C. Niemeyer and Oreste Panaia
}

The length of every pair $\{A, B\}$ of $n \times n$ complex matrices is at most $2 n-2$, if $n \leqslant 5$. That is, for $n \leqslant 5$, the (possibly empty) words in $A, B$ of length at most $2 n-2$ span the unital algebra $\mathcal{A}$ generated by $A, B$. For every positive integer $m$ there exist $m \times m$ complex matrices $C, D$ such that the length of the pair $\{C, D\}$ is $2 m-2$.

\section{INTRODUCTION AND PRELIMINARIES}

Let $\mathbb{F}$ be a field and let $M_{n}(\mathbb{F})$ denote the set of all $n \times n$ matrices over $\mathbb{F}$. Let $\mathcal{S}$ be a finite subset of $M_{n}(\mathbb{F})$. Let the identity matrix be defined to be the unique word in the alphabet $\mathcal{S}$ of length zero and also call it the empty word. For every positive integer $k$, define a word in the alphabet $\mathcal{S}$ to be of length $k$ if it has $k$ factors, counting multiplicities, so that, for example, the word $A^{2} B A C^{2} A^{3}$ has length 9 (assuming that $A, B, C \in \mathcal{S}$ ). For every natural number $k$ let $\mathcal{S}_{k}$ be the set of words in the alphabet $\mathcal{S}$ of length at most $k$ (including the empty word) and let $\mathcal{V}_{k}$ be the subspace of $M_{n}(\mathbb{F})$ spanned by $\mathcal{S}_{k}$. Clearly

$$
\mathbb{F} I=\mathcal{V}_{0} \subseteq \mathcal{V}_{1} \subseteq \mathcal{V}_{2} \subseteq \cdots \subseteq \mathcal{V}_{i} \subseteq \mathcal{V}_{i+1} \subseteq \cdots \subseteq \mathcal{A},
$$

where $\mathcal{A}$ is the unital algebra generated by $\mathcal{S}$. Since $\mathcal{A}$ is finite-dimensional, there is an integer $l$ such that $\mathcal{V}_{l}=\mathcal{V}_{l+1}$. Then $\mathcal{V}_{k}=\mathcal{V}_{l}$, for every $k>l$, since

$$
\mathcal{S}_{k} \subseteq \mathcal{S}_{k-l-1} \mathcal{S}_{l+1} \subseteq \mathcal{S}_{k-l-1} \mathcal{V}_{l} \subseteq \mathcal{V}_{k-1}
$$

so $\mathcal{V}_{k}=\mathcal{V}_{k-1}$, for such $k$. Since $\mathcal{A}=\bigcup_{k=0}^{\infty} \mathcal{V}_{k}$ we then have $\mathcal{A}=\mathcal{V}_{l}$. Following [3], we define the length $l(\mathcal{S})$ of $\mathcal{S}$ to be the smallest integer $l$ for which $\mathcal{V}_{l}=\mathcal{A}$. Then

$$
\mathbb{F} I=\mathcal{V}_{0} \subset \mathcal{V}_{1} \subset \mathcal{V}_{2} \subset \cdots \subset \mathcal{V}_{i} \subset \mathcal{V}_{i+1} \subset \cdots \subset \mathcal{V}_{l}=\mathcal{A}
$$

where ' $C$ ' denotes strict inclusion. From this we get the trivial upper bound $l(\mathcal{S})$ $\leqslant d-1$ where $d$ is the dimension of $\mathcal{A}$. (Similar types of upper bounds were observed in $[1,2,3,4,5]$.) In $[4]$ Paz conjectures that $l(\mathcal{S}) \leqslant 2 n-2$, and shows this to be the case Received 27th February, 2006

Copyright Clearance Centre, Inc. Serial-fee code: 0004-9727/06 \$A2.00+0.00. 
for $n \leqslant 4$, by proving that $l(\mathcal{S}) \leqslant\left\lceil\left(n^{2}+2\right) / 3\right\rceil$, whenever $n \geqslant 2$. (Here ' \lceil\rceil ' denotes the least integer function.) Examples exhibited or referred to in [4], attributed to J. W. Carlyle, show that the inequality $l(\mathcal{S}) \leqslant 2 n-2$ is sharp for $n=2,3$ or 4 , and that $l(\mathcal{S})=8$ can occur when $n=5$. Then [1, Example 2.9] shows that $l(\mathcal{S}) \geqslant 2 n-2$ for infinitely many values of $n$. Additionally, [3, Theorem 4.1 (b)] shows that $l(\mathcal{S}) \leqslant 2 n-2$ if $\mathcal{S}$ is a finite set of matrices which generates $M_{n}(\mathbb{F})$ as a unital algebra, and which contains a matrix with distinct eigenvalues. In [3] it is shown that $l(\mathcal{S}) \leqslant \sqrt{2} n^{3 / 2}+3 n$ for every $n$, for every finite set of matrices $\mathcal{S}$ which generates $M_{n}(\mathbb{F})$ as a unital algebra.

Here we shall be concerned with the case when $\mathbb{F}=\mathbb{C}$ and the alphabet $\mathcal{S}=\{A, B\}$ where $A$ and $B$ are $n \times n$ matrices. We show that $l(A, B) \leqslant 2 n-2$ for $n \leqslant 5$ and that the inequality is sharp for such $n$.

Let $\mathcal{S}$ be a finite set of $n \times n$ matrices over a field $\mathbb{F}$ which generates $M_{n}(\mathbb{F})$ as an algebra. Following [2] we define the minimum spanning length of $\mathcal{S}$, denoted $\operatorname{msl}(\mathcal{S})$, to be the smallest positive integer $m$ such that the set of nonempty words of length at most $m$, in the alphabet $\mathcal{S}$, span $M_{n}(\mathbb{F})$. It is obvious that $l(\mathcal{S}) \leqslant \operatorname{msl}(\mathcal{S})$. Almost as obvious is the fact that $\operatorname{msl}(\mathcal{S}) \leqslant l(\mathcal{S})+1$. (Note that if the nonempty words of length $l-1$ do not span $M_{n}(\mathbb{F})$ then their span is strictly included in the span of the nonempty words of length $l$.) We can have $l(\mathcal{S}) \neq \operatorname{msl}(\mathcal{S})$. Indeed, as is remarked in [2], if $B$ is the $3 \times 3$ complex strictly upper triangular elementary Jordan matrix and $A=B^{*}$, then $l(A, B)=3$ and $\operatorname{msl}(A, B)=4$.

\section{OTHER COMMENTS CONCERNING THE RESULTS OF [2].}

The manuscript for [2] was written in ignorance of $[1,3,4,5]$. The first author thanks Thomas J. Laffey for bringing these articles to his attention. In [2] only matrices over the complex field were considered, but some of the results apply to more general fields. Also, most of the results in [2] concerning 'minimum spanning length' yield results on 'length'.

1. Consideration of the proof of [2, Theorem 2] leads to the following theorem.

THEOREM 1. Let $\mathbb{F}$ be a field with characteristic zero. Let $n \geqslant 2$ and let $B \in M_{n}(\mathbb{F})$ be the strictly upper triangular elementary Jordan matrix. For any matrix $A \in M_{n}(\mathbb{F})$ such that $\{A, B\}$ generates $M_{n}(\mathbb{F})$ as an algebra, we have $m s l(A, B) \leqslant 2 n-2$.

2. With obvious modifications [2, Example 2], shows that, if $n \geqslant 3$ and $\mathbb{F}$ is a field with characteristic zero, then $\operatorname{msl}(A, B)=2 n-2$ where $B \in M_{n}(\mathbb{F})$ is the strictly upper triangular elementary Jordan matrix and $A=\left(B^{t}\right)^{n-1}$ (where $B^{t}$ denotes the transpose of $B$ ). In fact, since it shows that every word $W=\left(w_{i, j}\right)$ in $A$ and $B$, including the empty word, of length at most $2 n-3$ satisfies $w_{1, n-1}=w_{2, n}$ it follows that $l(A, B)=2 n-2$. The latter is true even when $n=2$.

3. The proofs of [2, Propositions 3 and 4] hold when the underlying field has characteristic zero, not just when it is $\mathbb{C}$. Thus we have the following proposition.

PROPOSITION 1. Let $\mathbb{F}$ be a field with characteristic zero. Let $n \geqslant 2, n \neq 3$, let 
$B \in M_{n}(\mathbb{F})$ be the strictly upper triangular elementary Jordan matrix and let $A=B^{t}$. Then $l(A, B)=\operatorname{msl}(A, B)=n$.

\section{MAIN RESUltS}

In the remainder of this paper the underlying field will be $\mathbb{C}$, the complex field. Let $n \geqslant 2$ and let $A, B$ be $n \times n$ complex matrices. Let $\mathcal{V}$ be the set of all words, including the empty word, in $A$ and $B$. If $U, V \in \mathcal{V}$ and $U$ and $V$ are the same word we write $U \equiv V$. (So $U \equiv V$ is strictly stronger than $U=V$ where the latter means equality as matrices.) For each integer $k \geqslant 1$, totally order the words in $A$ and $B$ of length $k$ using dictionary order. (So, if $W_{1}$ and $W_{2}$ are words of equal nonzero length, we say that $W_{1} \preceq W_{2}$ if $W_{1} \equiv X A V_{1}$ and $W_{2} \equiv X B V_{2}$, where each of $X, V_{1}, V_{2}$ is a word in $A, B$, possibly empty.) Extend these orders to a total order on $\mathcal{V}$ by additionally defining $W_{1} \preceq W_{2}$ if the length of $W_{1}$ is strictly less than the length of $W_{2}$.

In the totally ordered set $\mathcal{V}$, define $\mathcal{B}$ to be the set of elements which do not belong to the span of their strict predecessors. Then $\mathcal{B}$ is a linearly independent set of words, hence finite. Clearly $I \in \mathcal{B}$. Note that, if $W$ is a word in $\mathcal{B}$ of length at least 2 , then every proper subword of $W$ belongs to $\mathcal{B}$. For if a word $U$ belongs to the span of words strictly less than it so do the words $U V$ and $V U$, for any word $V$.

Now the length of the pair $\{A, B\}$ is at most $2 n-2$ if and only if $\mathcal{V}_{2 n-2}$ is the unital algebra generated by $A$ and $B$ if and only if $\mathcal{B}$ does not contain a word of length $2 n-1$. If $\mathcal{B}$ did contain a word of length $2 n-1$, we could let $W$ be the smallest word (in the sense of the total order $\preceq$ ) of length $2 n-1$ in $\mathcal{B}$. Then

(i) $W$ has length $2 n-1$,

(ii) $W$ has no factors of the form $A^{n}$ or $B^{n}$,

(iii) the number of subwords of $W$ (including the empty subword) is at most $n^{2}$.

We shall describe, for $2 \leqslant n \leqslant 5$ the forms that $W$ can have, given the constraints (i), (ii) and (iii) immediately above, and show that $W$ having any one of these forms leads to a contradiction. The pertinent question here is:

Question. Let $n \in \mathbb{Z}^{+}, n \geqslant 2$. Which words of length $2 n-1$ in the symbols $a$ and $b$ with no factor of $a^{n}$ or $b^{n}$ have at most $n^{2}$ subwords, including the empty subword?

Proposition 2. Let $n=2,3$ or 4 and let $w$ be a word of length $2 n-1$ in the symbols $a$ and $b$ with no factor of $a^{n}$ or $b^{n}$. If $n=2$ or $3, w$ has more than $n^{2}$ subwords. If $n=4, w$ has more than $n^{2}$ subwords unless it is $(a b)^{3} a$ or $(b a)^{3} b$ in which case it has 14 subwords.

Proof:

CASE $n=2$. If $n=2, w$ must be $a b a$ or $b a b$. Consequently, $w$ has $6>2^{2}$ subwords (These are $a b a, a b, b a, a, b, e$, where $e$ is the empty subword, if $w$ is $a b a$.) 
CASE $n=3$. If $n=3, w$ has length 5 . The words of length 5 with no factors of $a^{3}$ or $b^{3}$ are $a^{2} b a^{2}, a^{2} b a b, a^{2} b^{2} a, a b a^{2} b, a b a b a$ together with those 11 words that can be obtained from these 5 words by using symmetry, that is, by interchanging $a$ and $b$ or by reading them backwards or by combining the two. Now $a b a b a$ has 10 subwords and $a^{2} b^{2} a$ has 13 ; each of $a^{2} b a^{2}, a^{2} b a b, a b a^{2} b$ has 12 .

CASE $n=4$. If $n=4, w$ has length 7. If $w$ is either $(a b)^{3} a$ or $(b a)^{3} b$ it has 14 subwords. If it is neither of these words it has at least $17>4^{2}$ subwords. Indeed, the numbers of different subwords satisfy the following table.

\begin{tabular}{|c|c|c|c|c|c|c|c|c|}
\hline length & 7 & 6 & 5 & 4 & 3 & 2 & 1 & 0 \\
\hline \#subwords & 1 & 2 & 3 & $\geqslant 3$ & $\geqslant 2$ & $\geqslant 3$ & 2 & 1 \\
\hline
\end{tabular}

Note that if $w$ was $w_{1} w_{2} \ldots w_{7}$ where $w_{i} \in\{a, b\}, i=1,2, \ldots, 7$ and it had only 2 subwords of length 4 , then $w_{1} w_{2} w_{3} w_{4}$ and $w_{3} w_{4} w_{5} w_{6}$ would be the same, and so would $w_{2} w_{3} w_{4} w_{5}$ and $w_{4} w_{5} w_{6} w_{7}$ be. Consequently, $w$ would have the form $(p q)^{3} p$. It would also have this form if it had no factor of $a^{2}$ or $b^{2}$.

Corollary 1 . Let $n \in \mathbb{Z}$. If $n=2$ or 3 there are no words of length $2 n-1$ in $a$ and $b$ containing no factors of $a^{n}$ or $b^{n}$ with at most $n^{2}$ subwords. If $n=4$ the only words of length $2 n-1$ containing no factor of $a^{n}$ or $b^{n}$ with at most $n^{2}$ subwords (including the empty word) are $(a b)^{3} a$ and $(b a)^{3} b$. Each of the latter has 14 subwords.

Analysis of the case $n=5$ is a little more difficult.

PRoposition 3. Let $w$ be a word of length 9 in the letters $a$ and $b$ satisfying

(i) $w$ has no factor of the form $a^{5}$ or $b^{5}$,

(ii) $w$ is not of the form $(p q)^{4} r, p(q r)^{4}$ or $(p q r)^{3}$, where $\{p, q, r\} \subseteq\{a, b\}$.

Then $w$ has at least 26 subwords, including the empty subword.

ProOF: If words $f$ and $g$, in $a$ and $b$, are the same word we write $f \equiv g$.

Let $w \equiv w_{1} w_{2} \ldots w_{9}$ where $\left\{w_{1}, w_{2}, \ldots, w_{9}\right\} \subseteq\{a, b\}$. We shall show that the numbers of different subwords satisfy the following table.

\begin{tabular}{|c|c|c|c|c|c|c|c|c|c|c|}
\hline length & 9 & 8 & 7 & 6 & 5 & 4 & 3 & 2 & 1 & 0 \\
\hline \#subwords & 1 & 2 & 3 & 4 & $\geqslant 4$ & $\geqslant 4$ & $\geqslant 2$ & $\geqslant 3$ & 2 & 1 \\
\hline
\end{tabular}

The sum of the numbers in the last row is at least 26 , so this will prove the theorem.

Consider the 4 subwords of length 6 . Call them $V_{1} \equiv w_{1} w_{2} \ldots w_{6}, V_{2} \equiv w_{2} w_{3} \ldots w_{7}$, $V_{3} \equiv w_{3} w_{4} \ldots w_{8}, V_{4} \equiv w_{4} w_{5} \ldots w_{9}$. By condition (i), $V_{1} \not \equiv V_{2} \not \equiv V_{3} \not \equiv V_{4}$. If $V_{3} \equiv V_{1}$ then $w$ has the form $(p q)^{4} r$. If $V_{4} \equiv V_{2}$ then $w$ has the form $p(q r)^{4}$. If $V_{4} \equiv V_{1}$ then $w$ has the form $(p q r)^{3}$. Each of these contradicts condition (ii), so $V_{1}, V_{2}, V_{3}, V_{4}$ are distinct. It follows that all of the subwords of lengths $6,7,8$ or 9 are distinct. 
Consider the 5 subwords of length 5 . We shall abuse notation and call them $V_{1}, V_{2}, \ldots, V_{5}$, where

$V_{1} \equiv w_{1} w_{2} \ldots w_{5}, V_{2} \equiv w_{2} w_{3} \ldots w_{6}, V_{3} \equiv w_{3} w_{4} \ldots w_{7}, V_{4} \equiv w_{4} w_{5} \ldots w_{8}, V_{5} \equiv w_{5} w_{6} \ldots w_{9}$

Again, by condition (i), $V_{1} \not \equiv V_{2} \not \equiv V_{3} \not \equiv V_{4} \not \equiv V_{5}$. If at most 3 of these 5 subwords were distinct, there are only 7 possibilities, namely,

$$
\begin{aligned}
(5-3 i) & V_{1} \equiv V_{3} \text { and } V_{2} \equiv V_{5} \\
(5-3 \mathrm{ii}) & V_{1} \equiv V_{4} \text { and } V_{3} \equiv V_{5} \\
(5-3 i i i) & V_{1} \equiv V_{3} \text { and } V_{2} \equiv V_{4} \\
(5-3 i v) & V_{1} \equiv V_{3} \equiv V_{5} \\
(5-3 \mathrm{v}) & V_{1} \equiv V_{5} \text { and } V_{2} \equiv V_{4} \\
(5-3 v i) & V_{2} \equiv V_{4} \text { and } V_{3} \equiv V_{5} \\
(5-3 v i i) & V_{1} \equiv V_{4} \text { and } V_{2} \equiv V_{5}
\end{aligned}
$$

If case (5-3i) or (5-3ii) held, $w$ would be of the form $p^{9}$. If either of (5-3iii), (5-3iv) or (5-3v) held, $w$ would be of the form $(p q)^{4} r$. If (5-3vi) held, $w$ would be of the form $p(q r)^{4}$ and if (5-3vii) held it would be of the form $(p q r)^{3}$. Each of these contradicts condition (5-3i) or (5-3ii). Thus $w$ has at least 4 distinct subwords of length 5 .

Consider the 6 subwords of length 4 , namely, $V_{1} \equiv w_{1} \ldots w_{4}, V_{2} \equiv w_{2} \ldots w_{5}, V_{3} \equiv w_{3} \ldots w_{6}, V_{4} \equiv w_{4} \ldots w_{7}, V_{5} \equiv w_{5} \ldots w_{8}, V_{6} \equiv w_{6} \ldots w_{9}$ Again, by condition (i), $V_{1} \not \equiv V_{2} \not \equiv V_{3} \not \equiv V_{4} \not \equiv V_{5} \not \equiv V_{6}$. If at most 3 of these 6 subwords were distinct, there are only 15 possibilities, namely,

$$
\begin{aligned}
(4-3 \mathrm{i}) & V_{1} \equiv V_{3} \equiv V_{6} \text { and } V_{2} \equiv V_{4} \\
(4-3 \mathrm{ii}) & V_{1} \equiv V_{3} \equiv V_{6} \text { and } V_{2} \equiv V_{5} \\
(4-3 \mathrm{iii}) & V_{1} \equiv V_{3}, V_{2} \equiv V_{5} \text { and } V_{4} \equiv V_{6} \\
(4-3 \mathrm{iv}) & V_{1} \equiv V_{4} \equiv V_{6} \text { and } V_{2} \equiv V_{5} \\
(4-3 \mathrm{v}) & V_{1} \equiv V_{4} \equiv V_{6} \text { and } V_{3} \equiv V_{5} \\
(4-3 \mathrm{vi}) & V_{1} \equiv V_{4}, V_{2} \equiv V_{6} \text { and } V_{3} \equiv V_{5} \\
(4-3 \mathrm{vii}) & V_{1} \equiv V_{5}, V_{2} \equiv V_{4} \text { and } V_{3} \equiv V_{6} \\
(4-3 \mathrm{viii}) & V_{1} \equiv V_{6}, V_{2} \equiv V_{4} \text { and } V_{3} \equiv V_{5} \\
(4-3 \mathrm{ix}) & V_{1} \equiv V_{3} \equiv V_{5} \text { and } V_{2} \equiv V_{4} \\
(4-3 \mathrm{x}) & V_{1} \equiv V_{3} \text { and } V_{2} \equiv V_{4} \equiv V_{6} \\
(4-3 \mathrm{xi}) & V_{1} \equiv V_{3} \equiv V_{5} \text { and } V_{2} \equiv V_{6} \\
(4-3 \mathrm{xii}) & V_{1} \equiv V_{3} \equiv V_{5} \text { and } V_{4} \equiv V_{6} \\
(4-3 \mathrm{xiii}) & V_{1} \equiv V_{5} \text { and } V_{2} \equiv V_{4} \equiv V_{6} \\
(4-3 \mathrm{xiv}) & V_{1} \equiv V_{4} \equiv V_{6} \text { and } V_{3} \equiv V_{5}
\end{aligned}
$$


(4-3xv) $\quad V_{1} \equiv V_{4}, V_{2} \equiv V_{5}$, and $V_{3} \equiv V_{6}$.

If either of cases (4-3i) to (4-3viii) held, $w$ would be of the form $p^{9}$. If either of (4-3ix) to (4-3xiii) held, $w$ would be of the form $(p q)^{4} r$. If (4-3xiv) held, $w$ would be of the form $p(q r)^{4}$ and if (4-3xv) held it would be of the form $(p q r)^{3}$. But each of these contradicts condition (i) or (ii). Thus $w$ has at least 4 distinct subwords of length 4 .

The subwords $w_{1} w_{2} w_{3}, w_{2} w_{3} w_{4}, w_{3} w_{4} w_{5}$ of $w$ of length 3 cannot be the same, otherwise $w$ would be of the form $p^{5} q r s t$ which contradicts condition (i). Thus $w$ has at least two subwords of length 3 .

With regard to subwords of $w$ of length 2 , note that both $a b$ and $b a$ must be subwords by condition (i). Also, either $a^{2}$ or $b^{2}$ must be a subword, by condition (ii). Thus $w$ has at least 3 subwords of length 2 .

The above analysis shows that the subwords of $w$ occur as in the table given at the beginning of the proof. As remarked earlier, this completes the proof.

COROLlary 2. Let $w$ be a word in $a$ and $b$ of length 9. If $w$ has no factors of $a^{5}$ or $b^{5}$ and has 25 or fewer subwords (including the empty word) it must be one of

$$
\begin{gathered}
(a b)^{4} a ;(b a)^{4} b \\
\left(a^{2} b\right)^{3} ;\left(b^{2} a\right)^{3} ; a(a b)^{4} ; b(b a)^{4} ;(a b a)^{3} ;(b a b)^{3} ;(a b)^{4} b ;(b a)^{4} a ;\left(a b^{2}\right)^{3} ;\left(b a^{2}\right)^{3} .
\end{gathered}
$$

The words in the first row above each have 18 subwords; those in the last row have 24 .

Proof: By Proposition 3, $w$ must be of one of the forms $(p q)^{4} r, p(q r)^{4}$ or $(p q r)^{3}$, where $\{p, q, r\} \subseteq\{a, b\}$. Bearing in mind the fact that $w$ has no factor of $a^{5}$ or $b^{5}$ gives that it must be one of the 12 words listed. To verify that the actual number of subwords is as claimed, one need only consider, by symmetry, $(a b)^{4} a ;\left(a^{2} b\right)^{3} ; a(a b)^{4} ;(a b a)^{3}$.

Proposition 4. Let $n \geqslant 2$. For all complex $n \times n$ matrices $A$ and $B$, the matrix $(A B)^{n-1} A$ belongs to $\mathcal{V}_{2 n-3}$.

Proof: First, assume that $B$ is invertible. Let $W \equiv(A B)^{n-1} A$. Then, using the Cayley-Hamilton Theorem,

$$
W B \equiv(A B)^{n}=\lambda_{0} I+\sum_{k=1}^{n-1} \lambda_{k}(A B)^{k}
$$

for some scalars $\lambda_{0}, \lambda_{1}, \ldots, \lambda_{n-1}$. Hence

$$
W=\lambda_{0} B^{-1}+\sum_{k=1}^{n-1} \lambda_{k}(A B)^{k-1} A .
$$

Since $B^{-1}$ can be written as a polynomial in $B$ of degree $n-1$, the desired result follows.

Finally, assume that $B$ is not invertible. Choose a scalar $\lambda$ such that $B-\lambda I$ is invertible. By the above, $(A(B-\lambda I))^{n-1} A$ belongs to the span of words in $A, B-\lambda I$ of 
length at most $2 n-3$ together with the identity, and hence to $\mathcal{V}_{2 n-3}$. But

$$
(A(B-\lambda I))^{n-1} A-(A B)^{n-1} A
$$

belongs to $\mathcal{V}_{2 n-4}$, so the proof is complete.

Proposition 5. If $n=2,3$ or 4 , then $l(A, B) \leqslant 2 n-2$, for all $n \times n$ complex matrices $A$ and $B$, that is, the unital algebra generated by $A, B$ is the span of the set of all, possibly empty, words in $A, B$ of length at most $2 n-2$.

Proof: Define $\mathcal{B}$ as before, after totally-ordering the set of all words in $A$ and $B$. If $l(A, B)>2 n-2, \mathcal{B}$ would contain a word $W$ of length $2 n-1$. This word would have no factor of $A^{n}$ or $B^{n}$ and have at most $n^{2}$ subwords. By Corollary 1 , there is no such word if $n=2$ or 3 . If $n=4$, again by Corollary $1, W$ could only be $(A B)^{3} A$ or $(B A)^{3} B$. But these words cannot belong to $\mathcal{B}$ by Proposition 4 .

The remainder of this paper is devoted to showing that $n$ can also be taken to be 5 in Proposition 5.

TheOREM 2. For all $5 \times 5$ complex matrices $A$ and $B, l(A, B) \leqslant 8$.

Proof: Suppose that $l(A, B)>8$ and let $W$ be the smallest word of length 9 belonging to $\mathcal{B}$. Then $W$ contains no factors of $A^{5}$ or $B^{5}$ and has at most 25 subwords (including the empty subword). By Corollary 2 it must be one of the words

$$
\begin{aligned}
& (A B)^{4} A ;(B A)^{4} B ;\left(A^{2} B\right)^{3} ;\left(B^{2} A\right)^{3} ; A(A B)^{4} ; B(B A)^{4} ; \\
& (A B A)^{3} ;(B A B)^{3} ;(A B)^{4} B ;(B A)^{4} A ;\left(A B^{2}\right)^{3} ;\left(B A^{2}\right)^{3} .
\end{aligned}
$$

By Proposition 4, $W$ cannot be either $(A B)^{4} A$ or $(B A)^{4} B$. Now each of the remaining 10 possible words has 24 subwords. We complete the proof by showing that $W$ being any one of these 10 possible words leads to a contradiction. Notice that

(a) Since $W$ is the smallest word of length 9 belonging to $\mathcal{B}$, if $V$ is a word of length 9 satisfying $V \preceq W, V \not \equiv W$ then $V \in \mathcal{V}_{8}$,

(b) Since $M_{5}(\mathbb{C})$ has dimension 25 , every proper subword of any element of $\mathcal{B}$ is a subword of $W$.

By the Cayley-Hamilton Theorem both

$$
E=\frac{(A+B)^{5}+(A-B)^{5}}{2} \quad \text { and } \quad F=\frac{(A+i B)^{5}+(A-i B)^{5}}{2}
$$

belong to $\mathcal{V}_{4}$. Therefore, so do

(I) $Y=\frac{E-F}{2}=A^{3} B^{2}+A^{2} B A B+A^{2} B^{2} A+A B A^{2} B+A B A B A$

$$
+A B^{2} A^{2}+B A^{3} B+B A^{2} B A+B A B A^{2}+B^{2} A^{3} .
$$


and, interchanging $A$ and $B$,

$$
\begin{aligned}
Z=A^{2} B^{3}+A B A B^{2}+A B^{2} A B & +A B^{3} A+B A^{2} B^{2} \\
& +B A B A B+B A B^{2} A+B^{2} A^{2} B+B^{2} A B A+B^{3} A^{2}
\end{aligned}
$$

(i) Suppose that $W$ was the word $(A B)^{4} B$. In the expression for $Y$ the first 4 words on the right hand side are less than the fifth $A B A B A$. Thus, if $X$ is any of these first 4 words $(X) B A B^{2} \preceq(A B A B A) B A B^{2} \equiv W$ and so $(X) B A B^{2} \in \mathcal{V}_{8}$, by the remark (a) immediately above. Thus

(Y) $B A B^{2}=\left(A B A B A+A B^{2} A^{2}+B A^{3} B+B A^{2} B A+B A B A^{2}+B^{2} A^{3}\right) B A B^{2}+V \in \mathcal{V}_{8}$ where $V \in \mathcal{V}_{8}$. Hence $W+A B^{2} A^{2} B A B^{2}+B A^{3} B^{2} A B^{2}+B A^{2} B A B A B^{2}+B A B A^{2} B A B^{2}+B^{2} A^{3} B A B^{2} \in \mathcal{V}_{8}$

Neither of $A^{3}, A^{2} B$ belongs to $\mathcal{B}$, by remark (b) immediately above (each has $A^{2}$ as a proper subword), so both belong to $\mathcal{V}_{2}$ (because of the way that $\mathcal{B}$ is defined). Since each of

$$
A B^{2} A^{2} B A B^{2}, B A^{3} B^{2} A B^{2}, B A^{2} B A B A B^{2}, B A B A^{2} B A B^{2}, B^{2} A^{3} B A B^{2}
$$

has $A^{2} B$ as a factor, each of these 5 words belong to $\mathcal{V}_{8}$. It follows that $W \in \mathcal{V}_{8}$. This is a contradiction. Thus $W$ cannot be $(A B)^{4} B$.

The proof that $W$ cannot be $B(B A)^{4}$ is similar to the one just given.

(ii) Suppose that $W$ was the word $B(B A)^{4}$. In the expression (I) for $Y$, if $X$ is any of the first 4 words on the right hand side then $B^{2}(X) B A \preceq B^{2}(A B A B A) B A \equiv W$ and so $B^{2}(X) B A \in \mathcal{V}_{8}$. This gives

$B^{2}(Y) B A=B^{2}\left(A B A B A+A B^{2} A^{2}+B A^{3} B+B A^{2} B A+B A B A^{2}+B^{2} A^{3}\right) B A+V \in \mathcal{V}_{8}$ where $V \in \mathcal{V}_{8}$. Hence

$$
W+B^{2} A B^{2} A^{2} B A+B^{3} A^{3} B^{2} A+B^{3} A^{2} B A B A+B^{3} A B A^{2} B A+B^{4} A^{3} B A \in \mathcal{V}_{8}
$$

Again, neither $A^{3}$ nor $A^{2} B$ belongs to $\mathcal{B}$ so both belong to $\mathcal{V}_{2}$. Since each of

$$
B^{2} A B^{2} A^{2} B A, B^{3} A^{3} B^{2} A, B^{3} A^{2} B A B A, B^{3} A B A^{2} B A, B^{4} A^{3} B A
$$

has $A^{2} B$ as a factor, each of these 5 words belong to $\mathcal{V}_{8}$. It follows that $W \in \mathcal{V}_{8}$. This is a contradiction. Thus $W$ cannot be $B(B A)^{4}$.

(iii) Suppose that $W \equiv(B A)^{4} A$. If $X$ is any of the first 8 words on the right hand side in the expression (I) for $Y$, then $B A B A(X) \preceq B A B A\left(B A B A^{2}\right) \equiv W$ and so $B A B A(X) \in \mathcal{V}_{8}$. This gives

$$
B A B A(Y)=B A B A\left(B A B A^{2}+B^{2} A^{3}\right)+V_{1} \in \mathcal{V}_{8}
$$


where $V_{1} \in \mathcal{V}_{8}$. Hence $W+B A B A B^{2} A^{3} \in \mathcal{V}_{8}$. If $A^{3} \notin \mathcal{B}$ then $A^{3} \in \mathcal{V}_{2}$ and so $B A B A B^{2} A^{3} \in \mathcal{V}_{8}$. This gives $W \in \mathcal{V}_{8}$, which is a contradiction. Thus $A^{3} \in \mathcal{B}$, and the elements of $\mathcal{B}$ are precisely $A^{3}$ together with all the subwords of $W$. It follows that neither $A^{2} B$ nor $A B^{2}$ belongs to $\mathcal{B}$ and that $A B^{2}=\alpha A^{3}+\beta A B A+V_{2}$ for some scalars $\alpha, \beta$ and some element $V_{2} \in \mathcal{V}_{2}$. Hence

$$
B A B A B^{2} A^{3}=B A B\left(\alpha A^{3}+\beta A B A+V_{2}\right) A^{3}=\alpha B A B A^{6}+\beta B A B A B A^{4}+B A B V_{2} A^{3} .
$$

But $B A B A^{6} \in \mathcal{V}_{8}$ by the Cayley-Hamilton Theorem, $B A B A B A^{4} \in \mathcal{V}_{8}$ because $A^{4}$ $\in \mathcal{V}_{3}$ (since $A^{4} \notin \mathcal{B}$ ) and clearly $B A B V_{2} A^{3} \in \mathcal{V}_{8}$. Thus $B A B A B^{2} A^{3} \in \mathcal{V}_{8}$, so $W \in \mathcal{V}_{8}$. Again this is a contradiction.

(iv) Suppose that $W \equiv A(A B)^{4}$. If $X$ is any of the first 5 words on the right hand side in the expression (II) for $Z$, then $A^{2}(X) A B \preceq A^{2}(B A B A B) A B \equiv W$ and so $A^{2}(X) A B \in \mathcal{V}_{8}$. This gives

$$
A^{2}(Z) A B=A^{2}\left(B A B A B+B A B^{2} A+B^{2} A^{2} B+B^{2} A B A+B^{3} A^{2}\right) A B+V_{1} \in \mathcal{V}_{8}
$$

where $V_{1} \in \mathcal{V}_{8}$. Hence

$$
W+A^{2} B A B^{2} A^{2} B+A^{2} B^{2} A^{2} B A B+A^{2} B^{2} A B A^{2} B+A^{2} B^{3} A^{3} B \in \mathcal{V}_{8}
$$

None of $A^{4}, A^{3} B, A^{2} B^{2}, A B A^{2}, A B^{2}$ can belong to $\mathcal{B}$ since each has a proper subword which is not a subword of $W$. In particular, $A^{4}, A^{3} B \in \mathcal{V}_{3}$ and $A B^{2}=\alpha A^{3}$ $+\beta A^{2} B+\gamma A B A+V_{2}$ for some scalars $\alpha, \beta, \gamma$ and some $V_{2} \in \mathcal{V}_{2}$. Thus

$$
\begin{aligned}
A^{2} B\left(A B^{2}\right) A^{2} B & =A^{2} B\left(\alpha A^{3}+\beta A^{2} B+\gamma A B A+V_{2}\right) A^{2} B \\
& =\alpha A^{2} B A^{5} B+\beta\left(A^{2} B\right)^{3}+\gamma A^{2} B A B A^{3} B+A^{2} B V_{2} A^{2} B .
\end{aligned}
$$

Since $A B A^{2}=\delta A^{2} B A+V_{3}$ for some scalar $\delta$ and some $V_{3} \in \mathcal{V}_{3}$,

$$
\left(A^{2} B\right)^{3} \equiv A\left(A B A^{2}\right) B A^{2} B=A\left(\delta A^{2} B A+V_{3}\right) B A^{2} B=\delta A^{3} B A B A^{2} B+A V_{3} B A^{2} B \in \mathcal{V}_{8},
$$

since $A^{3} B \in \mathcal{V}_{3}$. Using the fact that $A^{3} B \in \mathcal{V}_{3}$ once again together with the CayleyHamilton Theorem gives that $A^{2} B A B^{2} A^{2} B \in \mathcal{V}_{8}$.

Finally note that

$$
\begin{aligned}
A^{2} B^{2} A^{2} B A B \equiv A\left(A B^{2}\right) A^{2} B A B=A\left(\alpha A^{3}+\beta A^{2} B+\gamma A B A+V_{2}\right) A^{2} B A B \\
=\alpha A^{6} B A B+\beta A^{3} B A^{2} B A B+\gamma A^{2} B A^{3} B A B+A V_{2} A^{2} B A B \in \mathcal{V}_{8}
\end{aligned}
$$

and

$$
\begin{aligned}
A^{2} B^{2} A B A^{2} B & \equiv A\left(A B^{2}\right) A B^{2} A B A^{2} B=A\left(\alpha A^{3}+\beta A^{2} B+\gamma A B A+V_{2}\right) A B A^{2} B \\
& =\alpha A^{5} B A^{2} B+\beta A^{3} B A B A^{2} B+\gamma\left(A^{2} B\right)^{3}+A V_{2} A B A^{2} B \in \mathcal{V}_{8}
\end{aligned}
$$


using the Cayley-Hamilton Theorem and the facts that $A^{3} B \in \mathcal{V}_{3},\left(A^{2} B\right)^{3} \in \mathcal{V}_{8}$. This now gives $W \in \mathcal{V}_{8}$ which is a contradiction. Thus $W$ cannot be of the form $A(A B)^{4}$.

(v) Suppose that $W$ was the word $\left(A^{2} B\right)^{3}$. In the expression (I) for $Y$, the first 7 words on the right hand side are less than the eighth $B A^{2} B A$. If $X$ is any one of these seven, $A^{2}(X) A B \preceq A^{2}\left(B A^{2} B A\right) A B \equiv W$, so $A^{2}(X) A B \in \mathcal{V}_{8}$. Thus

$$
A^{2}(Y) A B=A^{2}\left(B A^{2} B A+B A B A^{2}+B^{2} A^{3}\right) A B+V \in \mathcal{V}_{8}
$$

where $V \in \mathcal{V}_{8}$. Hence $W+A^{2} B A B A^{3} B+A^{2} B^{2} A^{4} B \in \mathcal{V}_{8}$. If $A^{3} \notin \mathcal{B}$ then $A^{3} \in \mathcal{V}_{2}$, so both $A^{2} B A B A^{3} B$ and $A^{2} B^{2} A^{4} B$ belong to $\mathcal{V}_{8}$, and it follows that $W \in \mathcal{V}_{8}$. This is a contradiction. Thus $A^{3} \in \mathcal{B}$ and $\mathcal{B}$ consists precisely of $A^{3}$ together with all the subwords of $W$. In particular, $A^{4}, A^{3} B \notin \mathcal{B}$ so $A^{4}, A^{3} B \in \mathcal{V}_{3}$. From the former it follows that $A^{2} B^{2} A^{4} B \in \mathcal{V}_{8}$ and from the latter that $A^{2} B A B A^{3} B \in \mathcal{V}_{8}$. It then follows that $W \in \mathcal{V}_{8}$. Again, this is a contradiction. Thus $W$ cannot be $\left(A^{2} B\right)^{3}$.

The proof that $W$ cannot be $(A B A)^{3}$ is similar to the above.

(vi) Suppose that $W \equiv(A B A)^{3}$. Note that if $X$ is any one of the first seven words on the right hand side in the expression (I) for $Y$, then

$$
A(X) A B A \preceq A\left(B A^{2} B A\right) A B A \equiv W,
$$

so $A(X) A B A \in \mathcal{V}_{8}$. It follows that $W+A B A B A^{3} B A+A B^{2} A^{4} B A \in \mathcal{V}_{8}$. Again, $A^{3}$ must belong to $\mathcal{B}$ and $A^{4}, A^{3} B \notin \mathcal{B}$, so $A^{4}, A^{3} B \in \mathcal{V}_{3}$. From the former it follows that $A B^{2} A^{4} B A \in \mathcal{V}_{8}$ and from the latter that $A B A B A^{3} B A \in \mathcal{V}_{8}$. It then follows that $W \in \mathcal{V}_{8}$. Again, this is a contradiction. Thus $W$ cannot be $(A B A)^{3}$.

(vii) Suppose that $W \equiv\left(B A^{2}\right)^{3}$. We use the same $Y$ and note that

$$
B A^{2}(X) A \preceq B A^{2}\left(B A^{2} B A\right) A \equiv W
$$

so $B A^{2}(X) A \in \mathcal{V}_{8}$, if $X$ is any one of the first seven words on the right hand side. This gives $W+B A^{2} B A B A^{3}+B A^{2} B^{2} A^{4} \in \mathcal{V}_{8}$ and again, $A^{3} \in \mathcal{B}$ and $\mathcal{B}$ consists precisely of $A^{3}$ together with all the subwords of $W$. In particular, $A^{4} \notin \mathcal{B}$ so $A^{4} \in \mathcal{V}_{3}$ and $B A^{2} B^{2} A^{4} \in \mathcal{V}_{8}$. Also (using the fact that $A B^{2} \notin B$ ) $B A B$ $=\alpha A^{3}+\beta A^{2} B+\gamma A B A+\delta B A^{2}+V$ for some scalars $\alpha, \beta, \gamma, \delta$ and some $V \in \mathcal{V}_{2}$. Then

$$
\begin{aligned}
B A^{2}(B A B) A^{3} & =B A^{2}\left(\alpha A^{3}+\beta A^{2} B+\gamma A B A+\delta B A^{2}+V\right) A^{3} \\
& =\alpha B A^{8}+\beta B A^{4} B A^{3}+\gamma B A^{3} B A^{4}+\delta B A^{2} B A^{5}+B A^{2} V A^{3} \in \mathcal{V}_{8}
\end{aligned}
$$

(since $A^{4} \notin \mathcal{B}$ implies that $A^{4} \in \mathcal{V}_{3}$ ). Again it follows that $W \in \mathcal{V}_{8}$. This is a contradiction so $W$ cannot be $\left(B A^{2}\right)^{3}$.

The proofs that $W$ cannot be $\left(A B^{2}\right)^{3},(B A B)^{3}$ or $\left(B^{2} A\right)^{3}$ are similar to one another. 
(viii) Suppose that $W \equiv\left(A B^{2}\right)^{3}$. In the expression (II) for $Z$ the first 6 words on the right hand side are less than the seventh $B A B^{2} A$. If $X$ is any one of these six, $A B(X) B^{2} \preceq A B\left(B A B^{2} A\right) B^{2} \equiv W$, so $A B(X) B^{2} \in \mathcal{V}_{8}$. Thus

$$
A B(Z) B^{2}=A B\left(B A B^{2} A+B^{2} A^{2} B+B^{2} A B A+B^{3} A^{2}\right) B^{2}+V \in \mathcal{V}_{8}
$$

where $V \in \mathcal{V}_{\mathbf{8}}$. Hence

$$
W+A B^{3} A^{2} B^{3}+A B^{3} A B A B^{2}+A B^{4} A^{2} B^{2} \in \mathcal{V}_{8} .
$$

Since $A B^{2} A$ is a subword of $W$ it belongs to $\mathcal{B}$. In fact $A B^{2} A$ is the smallest word of length 4 in $A$ and $B$ which belongs to $\mathcal{B}$. Indeed, none of $A^{4}, A^{3} B, A^{2} B A, A^{2} B^{2}, A B A^{2}$ can belong to $\mathcal{B}$ because each has $A^{2}$ as a proper subword, and $A^{2}$ is not a subword of $W$ (see remark (b) above). Also, $A B A B \notin \mathcal{B}$ since $A B A$ is not a subword of $W$. Thus, $A^{2} B^{2}$ and $A B A B$ both belong to $\mathcal{V}_{4}$. It follows that

$$
A B^{3} A^{2} B^{3}, A B^{3} A B A B^{2}, A B^{4} A^{2} B^{2} \in \mathcal{V}_{8}
$$

and that $W \in \mathcal{V}_{8}$. This is a contradiction so $W$ cannot be $\left(A B^{2}\right)^{3}$.

(ix) Suppose that $W \equiv(B A B)^{3}$. Using $Z$ as above, we have $(X) B^{2} A B$ $\preceq\left(B A B^{2} A\right) B^{2} A B \equiv W$, so $(X) B^{2} A B \in \mathcal{V}_{8}$, for any of the first 6 words $X$ on the right hand side of the expression (II) for $Z$. This gives

$$
W+B^{2} A^{2} B^{3} A B+B^{2} A B A B^{2} A B+B^{3} A^{2} B^{2} A B \in \mathcal{V}_{8}
$$

By exactly the same argument as in the proof of case (viii) immediately above, both $A^{2} B^{2}$ and $A B A B$ belong to $\mathcal{V}_{4}$. It follows that

$$
B^{2} A^{2} B^{3} A B, B^{2} A B A B^{2} A B, B^{3} A^{2} B^{2} A B \in \mathcal{V}_{8}
$$

and that $W \in \mathcal{V}_{8}$. Again, this is a contradiction so $W$ cannot be $(B A B)^{3}$.

(x) Suppose that $W \equiv\left(B^{2} A\right)^{3}$. Using $Z$ as above, we have $B(X) B^{2} A$ $\preceq B\left(B A B^{2} A\right) B^{2} A \equiv W$, so $B(X) B^{2} A \in \mathcal{V}_{8}$, for any of the first 6 words $X$ on the right hand side of the expression (II) for $Z$. This gives

$$
W+B^{3} A^{2} B^{3} A+B^{3} A B A B^{2} A+B^{4} A^{2} B^{2} A \in \mathcal{V}_{8} .
$$

Once again, both $A^{2} B^{2}$ and $A B A B$ belong to $\mathcal{V}_{4}$ so

$$
B^{3} A^{2} B^{3} A, B^{3} A B A B^{2} A, B^{4} A^{2} B^{2} A \in \mathcal{V}_{8}
$$

and $W \in \mathcal{V}_{8}$. This is a contradiction so $W$ cannot be $\left(B^{2} A\right)^{3}$.

This completes the proof of the theorem. 


\section{REFERENCES}

[1] A. Freedman, R. Gupta and R. Guralnick, 'Shirshov's theorem and representations of semigroups', Pacific J. Math., Special Issue (1997), 159-176.

[2] W.E. Longstaff, 'Burnside's Theorem: irreducible pairs of transformations', Linear Algebra Appl. 382 (2004), 247-269.

[3] C.J. Pappacena, 'An upper bound for the length of a finite-dimensional algebra', $J$. Algebra 197 (1997), 535-545.

[4] A. Paz, 'An application of the Cayley-Hamilton theorem to matrix polynomials in several variables', Linear and Multilinear Algebra 15 (1984), 161-170.

[5] C. Procesi, Rings with polynomial identities (Marcel Dekker, New York, 1973).

School of Mathematics and Statistics

The University of Western Australia

35 Stirling Highway

Crawley WA 6009

Australia

e-mail: longstaf@maths.uwa.edu.au

alice@maths.uwa.edu.au

oreste@maths.uwa.edu.au 